\title{
495.
}

\section{ON THE ENVELOPE OF A CERTAIN QUADRIC SURFACE.}

[From the Quarterly Journal of Pure and Applied Mathematics, vol. xI. (1871), pp. $244-246$.]

To find the envelope of the quadric surface

$$
a x^{2}+b y^{2}+c z^{2}+d w^{2}=0
$$

where the coefficients vary subject to the conditions -

$$
\left\{\begin{array}{l}
a \alpha^{2}+b \beta^{2}+c \gamma^{2}+d \delta^{2}=0 \\
\frac{p^{2}}{a}+\frac{q^{2}}{b}+\frac{r^{2}}{c}+\frac{s^{2}}{d}=0
\end{array}\right.
$$

$(\alpha, \beta, \gamma, \delta)$ and $(p, q, r, s)$ being respectively constant.

We have in the usual manner

$$
\begin{aligned}
& x^{2}+\lambda \alpha^{2}+\mu \frac{p^{2}}{a^{2}}=0 \\
& y^{2}+\lambda \beta^{2}+\mu \frac{q^{2}}{b^{2}}=0 \\
& z^{2}+\lambda \gamma^{2}+\mu \frac{r^{2}}{c^{2}}=0 \\
& w^{2}+\lambda \delta^{2}+\mu \frac{s^{2}}{d^{2}}=0
\end{aligned}
$$


and thence $a^{2}=\frac{-\mu p^{2}}{x^{2}+\lambda \alpha^{2}}$, \&c., and substituting these values $\mu$ disappears and we have

$$
\begin{gathered}
p \sqrt{ }\left(x^{2}+\lambda \alpha^{2}\right)+q \sqrt{ }\left(y^{2}+\lambda \beta^{2}\right)+r \sqrt{ }\left(z^{2}+\lambda \gamma^{2}\right)+s \sqrt{ }\left(w^{2}+\lambda \delta^{2}\right)=0, \\
\frac{\alpha^{2} p}{\sqrt{\left(x^{2}+\lambda \alpha^{2}\right)}}+\frac{\beta^{2} q}{\sqrt{\left(y^{2}+\lambda \beta^{2}\right)}}+\frac{\gamma^{2} r}{\sqrt{\left(z^{2}+\lambda \gamma^{2}\right)}}+\frac{\delta^{2} s}{\sqrt{\left(w^{2}+\lambda \delta^{2}\right)}}=0,
\end{gathered}
$$

from which $\lambda$ is to be eliminated; the second equation is here the derived function of the first in regard to $\lambda$, so that rationalising the first equation, the result is, as will be shown, of the form $(* \gamma \lambda, 1)^{4}=0$, and the result is obtained by equating to zero the discriminant of the quartic function.

Denoting for shortness the first equation by

$$
A+B+C+D=0
$$

the rationalised form is

$$
\left(A^{4}+B^{4}+C^{4}+D^{4}-2 A^{2} B^{2}-2 A^{2} C^{2}-2 A^{2} D^{2}-2 B^{2} C^{2}-2 B^{2} D^{2}-2 C^{2} D^{2}\right)^{2}-64 A^{2} B^{2} C^{2} D^{2}=0,
$$

which is of the form

where

$$
-\left(\mathfrak{A}+2 \mathfrak{B} \lambda+\left(\mathfrak{S} \lambda^{2}\right)^{2}+(a, b, c, d, e \gamma 1, \lambda)^{4}=0,\right.
$$

$$
\begin{aligned}
\mathfrak{A} & =p^{4} x^{4} \ldots-2 p^{2} q^{2} x^{2} y^{2} \ldots, \\
\mathfrak{B} & =p^{4} \alpha^{2} x^{2} \ldots-p^{2} q^{2}\left(\alpha^{2} y^{2}+\beta^{2} x^{2}\right) \ldots, \\
\mathfrak{S} & =p^{4} \alpha^{4} \ldots-2 p^{2} q^{2} \alpha^{2} \beta^{2} \ldots \\
\mathrm{a} & =8 \cdot x^{2} y^{2} z^{2} w^{2} \\
4 \mathrm{~b} & =8 \cdot \alpha^{2} y^{2} z^{2} w^{2}+\ldots \\
6 \mathrm{c} & =8 \cdot \alpha^{2} \beta^{2} z^{2} w^{2}+\ldots \\
4 \mathrm{~d} & =8 \cdot \alpha^{2} \beta^{2} \gamma^{2} w^{2}+\ldots \\
\mathrm{e} & =8 \cdot \alpha^{2} \beta^{2} \gamma^{2} \delta^{2} .
\end{aligned}
$$

Writing $I^{\prime}, J^{\prime}$ for the two invariants we find without difficulty

where

$$
\begin{aligned}
& I^{\prime}=I-\frac{4}{3} P+\Delta^{2}, \\
& J^{\prime}=J-Q+\frac{1}{3} \Delta P-\frac{8}{27} \Delta^{3},
\end{aligned}
$$

$$
\begin{aligned}
& I=\mathrm{ae}-4 \mathrm{bd}+3 \mathrm{c}^{2}, \\
& J=a c e-a d^{2}-b^{2} e-c^{3}+2 b c d, \\
& \Delta=\mathfrak{A}\left(\mathfrak{S}-\mathfrak{B}^{2},\right. \\
& P=\mathrm{a}^{2} \mathfrak{C}^{2}-4 \mathrm{~b} \mathfrak{B} \mathfrak{C}+2 \mathrm{c}\left(\mathfrak{A C}\left(\mathfrak{C}+2 \mathfrak{B}^{2}\right)-4 \mathrm{~d} \mathfrak{A} \mathfrak{B}+\mathrm{e}^{\mathfrak{2}},\right. \\
& Q=\left(c e-d^{2}\right) \mathfrak{A}^{2}+\left(a e+2 b d-3 c^{2}\right) \cdot \frac{1}{3}\left(\mathfrak{A C} \mathfrak{C}+2 \mathfrak{B}^{2}\right)+\left(a c-b^{2}\right)\left(\mathfrak{\complement}^{2}\right. \\
& -2(\mathrm{ad}-\mathrm{bc}) \mathfrak{B}(5
\end{aligned}
$$

C. VIII. 
The result thus is

$$
\left(I-P+\frac{4}{3} \Delta^{2}\right)^{3}-27\left(J-Q+\frac{1}{3} \Delta P-\frac{8}{27} \Delta^{3}\right)^{2}=0,
$$

or, what is the same thing, it is

$$
\begin{aligned}
(I-P)^{3}-27(J-Q)^{2} & -9 \Delta P(J-2 Q) \\
& +\Delta^{2}\left(4 I^{2}-8 I P+P^{2}\right) \\
& +8 \Delta^{3}(J-2 Q) \\
& +\Delta^{4} \cdot \frac{16}{3} I=0
\end{aligned}
$$

where the left-hand side is of the order 24 in $(x, y, z, w)$. I apprehend that the order should be $=12$ only; for writing $(x, y, z, w)$ in place of $\left(x^{2}, y^{2}, z^{2}, w^{2}\right)$, the equations which connect $(a, b, c, d)$ express that these quantities are the coordinates of a point on a plane cubic; and the problem is in fact that of finding the reciprocal of the plane cubic: this is a sextic cone, or restoring $\left(x^{2}, y^{2}, z^{2}, w^{2}\right)$ instead of $(x, y, z, w)$, we should have a surface of the order 12. I cannot explain how the reduction is effected. 